\title{
Reconstructing Stress Resultants in Wind Turbine Towers Based on Strain Measurements
}

\author{
Marko Kinne ${ }^{1(\bigotimes)}$, Ronald Schneider ${ }^{1}$, and Sebastian Thöns ${ }^{1,2}$ \\ ${ }^{1}$ Bundesanstalt für Materialforschung und-prüfung (BAM), Berlin, Germany \\ marko.kinne@bam.de \\ 2 Lund University, Lund, Sweden
}

\begin{abstract}
Support structures of offshore wind turbines are subject to cyclic stresses generated by different time-variant random loadings such as wind, waves, and currents in combination with the excitation by the rotor. In the design phase, the cyclic demand on wind turbine support structure is calculated and forecasted with semi or fully probabilistic engineering models. In some cases, additional cyclic stresses may be induced by construction deviations, unbalanced rotor masses and structural dynamic phenomena such as, for example, the Sommerfeld effect. Both, the significant uncertainties in the design and a validation of absence of unforeseen adverse dynamic phenomena necessitate the employment of measurement systems on the support structures. The quality of the measurements of the cyclic demand on the support structures depends on (a) the precision of the measurement system consisting of sensors, amplifier and data normalization and (b) algorithms for analyzing and converting data to structural health information. This paper presents the probabilistic modelling and analysis of uncertainties in strain measurements performed for the purposes of reconstructing stress resultants in wind turbine towers. It is shown how the uncertainties in the strain measurements affect the uncertainty in the individual components of the reconstructed forces and moments. The analysis identifies the components of the vector of stress resultants that can be reconstructed with sufficient precision.
\end{abstract}

Keywords: Strain measurements - Bayesian updating of measurement uncertainties $\cdot$ Reconstruction of stress resultants

\section{Introduction}

The main components of a fixed offshore wind turbine are the rotor including the blades, the nacelle housing the generator, the tower, and the support structure [1]. The tower and the support structure are connected via a transition piece. In the design, the timedependent behavior of a wind turbine system is determined using models describing the relevant physics and the turbine control system. Even though the models are highly sophisticated, they are subject to uncertainty. On the one hand, uncertainty is present in the model parameters. On the other hand, uncertainty is present in the models themselves (e.g. model uncertainty) as they do not necessarily include all influencing factors and/or processes. To reduce the uncertainty in the modelling and consequently in the estimates 
of the performance, operators can install monitoring systems to obtain data from the real turbine system. As an example, strain gauge rosettes can be applied at different elevations of the turbine tower to monitor its actual strain and stress state. If the rosettes are positioned appropriately around the circumference of a turbine tower, the actual timedependent stress resultants (forces and moments) in this cross-section can be monitored. These load effects can, for example, be applied to updated predictions of the system's fatigue performance and improve decisions on inspection and maintenance actions as well as support decisions on a lifetime extension.

In this contribution, an approach to reconstruct stress resultants in a turbine tower cross-section from measured strains is presented. In this approach, a prior estimate of the uncertainty in strain measurements is determined based on a physical model of the measurement process [2-4]. Subsequently, the prior measurement uncertainty is updated with outcomes of actual strain measurements using Bayesian updating [2-4]. The updated measurement uncertainties together with material and modelling uncertainties are then considered in the reconstruction of the stress results. Their impact on the reconstructed stress resultants is investigated in a numerical example.

\section{Modelling Uncertainty in Strain Measurements}

\subsection{Prior Probabilistic Model of the Measurement Uncertainty}

Mechanical strains can be measured with strain gauges. Strain gauge configurations are commonly based on the Wheatstone bridge concept, through which small changes in electrical resistance can be measured. In a quarter-bridge configuration with one active strain gauge, an amplifier supplies voltage $U_{B}[\mathrm{~V}]$ to the bridge circuit, amplifies the corresponding bridge output voltage $U_{A}[\mathrm{mV}]$ and determines the $i$ th measured strain $\varepsilon_{M}[\mu \mathrm{m} / \mathrm{m}]$ based on the following model [5]:

$$
\varepsilon_{M}=\frac{4}{k} \frac{U_{A}}{U_{B}}
$$

where $k[-]$ is the batch-specific gauge factor provided by the manufacturer.

Strain measurements are subject to various uncertain influencing factors and hence the measured strain $\varepsilon_{M}$ is not identical to the true mechanical strain $\varepsilon[\mu \mathrm{m} / \mathrm{m}]-$ the measurand. In the following, the relation between $\varepsilon$ and $\varepsilon_{M}$ is modelled by the following process Eq. (2):

$$
\varepsilon=B_{p}+\frac{f_{a a}}{c_{k}\left(\mathbf{X}_{k}, T\right)} \varepsilon_{M}+f_{a z}+\varepsilon_{T}\left(\mathbf{X}_{T}, T\right)
$$

wherein $B_{p}[\mu \mathrm{m} / \mathrm{m}]$ describes the model uncertainty; $f_{a a}$ [-] is the uncertain amplifying deviation factor; $f_{a z}[\mu \mathrm{m} / \mathrm{m}]$ is the uncertain amplifier zero deviation and $T\left[{ }^{\circ} \mathrm{C}\right]$ is temperature of the substrate, which is also measured. (Note that the uncertainty in the measured temperatures is neglected in the following. Also note that the values of $f_{a a}$ and $f_{a z}$ depend on the measured value $\varepsilon_{M}$ [2].) $c_{k}\left(\mathbf{X}_{k}, T\right)[-]$ is the correction coefficient of the gauge factor $k$, which is defined as [2]:

$$
c_{k}\left(\mathbf{X}_{k}, T\right)=1+B_{s}+f_{s, v}+f_{s, q}+\alpha_{k} \cdot\left(T-20^{\circ} \mathrm{C}\right)
$$


where $B_{s}[-]$ quantifies the model uncertainty associated with the gauge factor correction model; $f_{s, v}[-]$ is the gauge factor variation; $f_{s, q}[-]$ is the transverse strain correction factor and $\alpha_{k} \cdot\left(T-20^{\circ} \mathrm{C}\right)[-]$ models the temperature variation of the gauge factor. The coefficient $\alpha_{k}[1 / \mathrm{K}]$ is an empirical quantity. The transverse strain correction factor $f_{s, q}$ is given by [5]:

$$
f_{s, q}=\frac{q}{1-q v_{0}}\left(\frac{\varepsilon_{q}}{\varepsilon_{l}}+v_{0}\right)
$$

wherein $q[-]$ is the transverse sensitivity; $v_{0}[-]$ is Poisson's ratio of the material used in the experiments performed by the manufacture to determine the gauge factor and $\varepsilon_{q}$ $[\mu \mathrm{m} / \mathrm{m}]$ and $\varepsilon_{l}[\mu \mathrm{m} / \mathrm{m}]$ are the actual strains perpendicular and parallel to the primary axis of the strain gauge. In Eq. (2), $\varepsilon_{T}\left(\mathbf{X}_{T}, T\right)[\mu \mathrm{m} / \mathrm{m}]$ is introduced to computationally compensate the temperature drift of the sensor. This quantity is referred to as the apparent strain and is defined as:

$$
\varepsilon_{T}\left(\mathbf{X}_{T}, T\right)=\hat{\varepsilon}_{T}(T)+B_{T} \cdot\left(T-20^{\circ} \mathrm{C}\right)
$$

where $\hat{\varepsilon}_{T}(T)[\mu \mathrm{m} / \mathrm{m}]$ is a batch-specific temperature-variation curve supplied by the manufacturer and $B_{T} \cdot\left(T-20^{\circ} \mathrm{C}\right)[\mu \mathrm{m} / \mathrm{m}]$ is the model uncertainty of the temperaturevariation curve.

The uncertain parameters of the process equation $\mathbf{X}=\left[B_{p}, f_{a a}, f_{a z}, \mathbf{X}_{k}^{\mathrm{T}}, \mathbf{X}_{T}^{T}\right]^{T}$ with $\mathbf{X}_{k}=\left[B_{s}, f_{s, v}, \alpha_{k}\right]^{T}$ and $\mathbf{X}_{T}=\left[B_{T}\right]$ are modelled probabilistically [2], i.e. they are modelled as random variables. Their joint probability density function (PDF) is denoted by $p(\mathbf{x})$.

The process equation defined in Eq. (2) combined with the probabilistic model $p(\mathbf{x})$ of its parameters $\mathbf{X}$ constitutes the prior probabilistic model of the measurement uncertainty [2]. Based on this model, the prior distribution of the mechanical strain $p^{\prime}\left(\varepsilon \mid \varepsilon_{M}, T\right)$ in function of the measurement $\varepsilon_{M}$ and the temperature $T$ is constructed using a Monte Carlo (MC) approach [2]. To this end, samples of the mechanical strain $\varepsilon$ are generated by propagating the uncertainties in the parameters $\mathbf{X}$ through the process equation while the values of $\varepsilon_{M}$ and $T$ are kept fixed. Based on the samples of $\varepsilon$, a probabilistic model of $\varepsilon$ is fitted (e.g. a normal distribution) [2]. $p^{\prime}\left(\varepsilon \mid \varepsilon_{M}, T\right)$ quantifies the prior uncertainty in the measurand $\varepsilon$.

\subsection{Bayesian Updating of the Measurement Uncertainty}

The prior probabilistic model of the measurand $p^{\prime}\left(\varepsilon \mid \varepsilon_{M}, T\right)$ determined based on the prior probabilistic process equation (Eq. (2)) is updated with actual strain measurements using a Bayesian approach. To this end, controlled experiments are performed. In each experiment, the true mechanical strain $\varepsilon$ and the temperature $T$ are kept fixed and a series of $n$ strain measurements $\left\{\varepsilon_{M, i}\right\}_{i=1}^{n}$ is recorded with the measurement system modelled by the process equation. Based on the recorded strains, a likelihood function $L\left(\varepsilon \mid \varepsilon_{M}, T\right) \propto p\left(\varepsilon_{M} \mid \varepsilon, T\right)$ describing the measurement outcome is constructed.

It is assumed, that the relation between the true strain $\varepsilon$ and the measured strain $\varepsilon_{M}$ can be described by an additive measurement error $e$, i.e.

$$
\varepsilon_{M}=\varepsilon+e
$$


The probabilistic distribution of $e$ at temperature $T$ is denoted by $p(e \mid T)$. In the following, it is assumed that the measurement error $e$ is normal distributed with zero mean and standard deviation $\sigma_{e}$. From Eq. (6), it follows that:

$$
e=\varepsilon_{M}-\varepsilon
$$

When the value of $\varepsilon$ is known, the distribution of $e$ corresponds to the distribution of $\varepsilon_{M}$ shifted by $\varepsilon$. It follows, that for given $\varepsilon$ the standard deviation of $e$ is equal to the standard deviation of $\varepsilon_{M}$, which can be determined from the recorded strain measurements $\left\{\varepsilon_{M, i}\right\}_{i=1}^{n}$.

Once the parameters of the distribution $p(e \mid T)$ are known (i.e. the standard deviation $\left.\sigma_{e}\right)$, the likelihood function $L\left(\varepsilon \mid \varepsilon_{M}, T\right) \propto p\left(\varepsilon_{M} \mid \varepsilon, T\right)$ can be formulated as [6]:

$$
L\left(\varepsilon \mid \varepsilon_{M}, T\right)=p\left(e=\varepsilon_{M}-\varepsilon \mid T\right)
$$

Subsequently, the prior distribution $p^{\prime}\left(\varepsilon \mid \varepsilon_{M}, T\right)$ of the measurand $\varepsilon$ can be updated using Bayes' theorem:

$$
p^{\prime \prime}\left(\varepsilon \mid \varepsilon_{M}, T\right)=\frac{L\left(\varepsilon \mid \varepsilon_{M}, T\right) \cdot p^{\prime}\left(\varepsilon \mid \varepsilon_{M}, T\right)}{\int_{-\infty}^{\infty} L\left(\varepsilon \mid \varepsilon_{M}, T\right) \cdot p^{\prime}\left(\varepsilon \mid \varepsilon_{M}, T\right) d \varepsilon}
$$

where $p^{\prime \prime}\left(\varepsilon \mid \varepsilon_{M}, T\right)$ is the posterior (updated) distribution of the measurand $\varepsilon$, which quantifies the updated measurement uncertainty. $p^{\prime \prime}\left(\varepsilon \mid \varepsilon_{M}, T\right)$ depends on the measured strain $\varepsilon_{M}$ and the temperature $T$ and must be separately derived for each combination of the measured strain $\varepsilon_{M}$ and temperature $T$ [2].

\section{Reconstructing Stress Resultants in Wind Turbine Towers}

\subsection{Inverse Mechanical Model (Relating a Strain State to Stress Resultants)}

Figure 1(a) illustrates a cross-section of a tubular steel wind turbine tower. The blue coordinate system in Fig. 1(a) with origin at the center of the circular cross-section and coordinates $(x, y, z)$ is the global reference coordinate system. Applying classical beam theory, the stress resultants in this cross-section consisting of axial force $N_{x}$, shear forces $V_{y}$ and $V_{z}$, bending moments $M_{y}$ and $M_{z}$, and torsional moment $M_{x}$ can be reconstructed from strains measured by three strain rosettes $i=1,2,3$ distributed evenly around the circumference of the section. The strain rosettes indicated as red dots in Fig. 1(a) are here assumed to be applied to the outer surface of the tower. Each rosette consists of three strain gauges $a, b$ and $c$ as illustrated in Fig. 1(b). At the position of each rosette $i=1,2,3$, a local coordinate system with coordinates $\left(\xi_{i}, \eta_{i}\right)$ is introduced. The $\xi_{i}$-axis points in tangential direction of the outer surface of the tube and the $\eta_{i}$-axis is aligned with the global $x$-axis. Figure 2 shows a top view of the cross-section including the positions of the three strain rosettes and the global and local coordinate systems.

It can be shown that the mechanical strains $\varepsilon_{a, i}, \varepsilon_{b, i}$ and $\varepsilon_{c, i}$ at position $i$ can be transformed to the normal strains $\varepsilon_{\eta, i}$ and $\varepsilon_{\xi, i}$ and the shear strain $\psi_{\eta \xi, i}$ of an infinitesimal material element illustrated in Fig. 1(b) as follows:

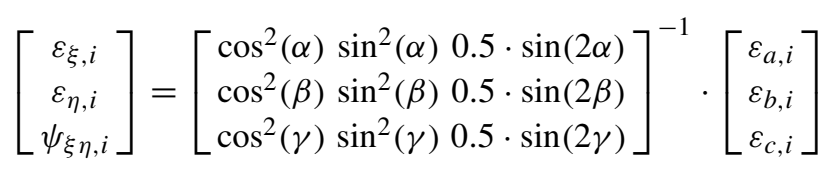


(a)
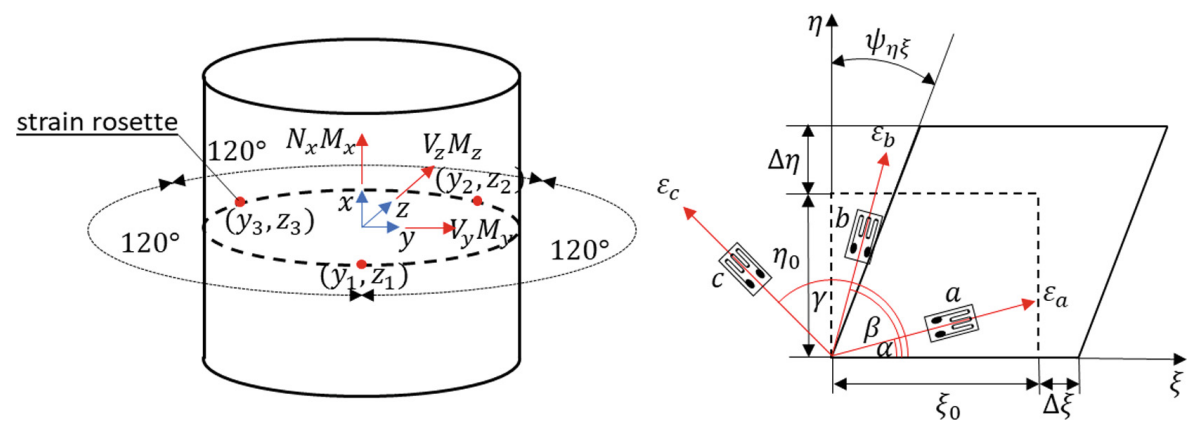

Fig. 1. (a) Cross-section along a tubular steel wind turbine tower with three strain rosettes positioned around the section's circumference and (b) illustration of the strain rosette on the surface of the tube consisting of three strain gauges $a, b$ and $c$.

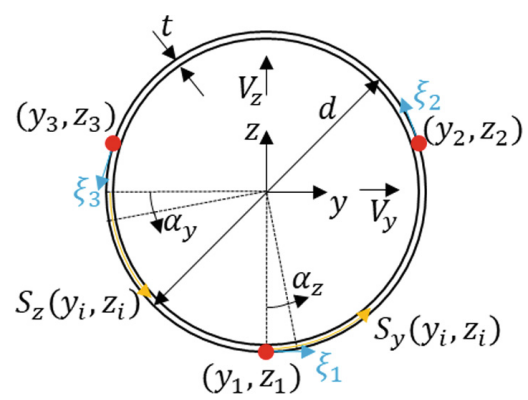

Fig. 2. Top view of the cross-section including the positions of the three strain rosettes and the global coordinate system with coordinates $(x, y, z)$ and local coordinate systems with coordinates $\left(\xi_{i}, \eta_{i}\right), i=1,2,3$. The local $\eta_{i}$-axis points in the same direction as the global $x$-axis.

The angles $\alpha, \beta$ and $\gamma$ determine the orientation of the strain gauges (see Fig. 1(b)).

Assuming linear-elastic material behavior and a plane stress state, the normal stresses $\sigma_{\xi, i}$ and $\sigma_{\eta, i}$ and the shear stress $\tau_{i}$ at position $i$ corresponding to $\varepsilon_{\xi, i}, \varepsilon_{\eta, i}$ and $\psi_{\xi \eta, i}$ can be determined as:

$$
\left[\begin{array}{c}
\sigma_{\xi, i} \\
\sigma_{\eta, i} \\
\tau_{i}
\end{array}\right]=\frac{E}{1-v^{2}}\left[\begin{array}{ccc}
1 & v & 0 \\
v & 1 & 0 \\
0 & 0 & 0.5 \cdot(1-v)
\end{array}\right] \cdot\left[\begin{array}{c}
\varepsilon_{\xi, i} \\
\varepsilon_{\eta, i} \\
2 \psi_{\xi \eta, i}
\end{array}\right]
$$

where $E$ and $v$ are the Young's modulus and Poisson's ratio of steel.

Applying classical beam theory and noting that $\sigma_{x}\left(y_{i}, z_{i}\right)=\sigma_{\eta, i}$, the relation between the normal stress $\sigma_{x}\left(y_{i}, z_{i}\right)$ at position $i$ with global coordinates $\left(y_{i}, z_{i}\right)$ and the section force and moments $N_{x}, M_{y}$ and $M_{z}$ can be written as:

$$
\sigma_{x}\left(y_{i}, z_{i}\right)=\sigma_{\eta, i}=\frac{N_{x}}{A}+\frac{M_{y}}{I_{y}} z_{i}-\frac{M_{z}}{I_{z}} y_{i}
$$


where $A$ is the cross-sectional area of the thin-walled tubular cross-section and $I_{y}$ and $I_{z}$ are the corresponding moment of inertia about $y$ and $z$-axis. Since the normal stress $\sigma_{x}\left(y_{i}, z_{i}\right)=\sigma_{\eta, i}$ can be determined at each position $i=1,2,3$ from the measured strains $\varepsilon_{a, i}, \varepsilon_{b, i}$ and $\varepsilon_{c, i}$, it is possible to formulate a system of linear equations with unknown $N_{x}, M_{y}$ and $M_{z}$ based on Eq. (12):

$$
\left[\begin{array}{l}
1 / A \\
z_{1} / I_{Y}-\mathrm{y}_{1} / I_{z} \\
1 / \mathrm{A} z_{2} / I_{Y}-\mathrm{y}_{2} / I_{z} \\
1 / \mathrm{A} z_{3} / I_{Y}-\mathrm{y}_{3} / I_{z}
\end{array}\right] \cdot\left[\begin{array}{l}
N_{x} \\
M_{y} \\
M_{z}
\end{array}\right]=\left[\begin{array}{l}
\sigma_{\eta, 1} \\
\sigma_{\eta, 2} \\
\sigma_{\eta, 3}
\end{array}\right]=\left[\begin{array}{l}
\sigma_{x}\left(y_{1}, z_{1}\right) \\
\sigma_{x}\left(y_{2}, z_{2}\right) \\
\sigma_{x}\left(y_{3}, z_{3}\right)
\end{array}\right]
$$

This system of linear equations can be solved for $N_{x}, M_{y}$ and $M_{z}$.

The shear stress $\tau\left(y_{i}, z_{i}\right)$ at position $i$ can be expressed in terms of the shear forces $V_{y}$ and $V_{z}$ and the torsional moment $M_{x}$ as:

$$
\tau\left(y_{i}, z_{i}\right)=\frac{M_{x}}{2 A_{m} t}-\frac{V_{y} S_{z}\left(y_{i}, z_{i}\right)}{I_{z} t}-\frac{V_{z} S_{y}\left(y_{i}, z_{i}\right)}{I_{y} t}
$$

with

$$
\begin{aligned}
& S_{y}\left(y_{i}, z_{i}\right)=-\int_{0}^{\alpha_{z}\left(y_{i}, z_{i}\right)}\left(\frac{d-t}{2}\right)^{2} t \cos \left(\alpha_{z}\right) d \alpha_{z} \\
& S_{z}\left(y_{i}, z_{i}\right)=-\int_{0}^{\alpha_{y}\left(y_{i}, z_{i}\right)}\left(\frac{d-t}{2}\right)^{2} t \cos \left(\alpha_{y}\right) d \alpha_{y}
\end{aligned}
$$

where $A_{m}=0.25 \pi(d-t), d$ is the outer diameter of the tubular cross-section, $t$ is the wall thickness, and $S_{y}\left(y_{i}, z_{i}\right)$ and $S_{z}\left(y_{i}, z_{i}\right)$ are the first moments of area at position $i$ in $y$ and $z$-direction. The angles $\alpha_{y}$ and $\alpha_{z}$ in Eq. (15) are defined in Fig. 2. Note that the relation between $\tau\left(y_{i}, z_{i}\right)$ and $M_{x}$ is based on Bredt's formula [7].

Based on Eq. (14), it is now possible to formulate a linear system of equation that relates the shear forces $V_{y}$ and $V_{z}$ and the torsional moment $M_{x}$ to the shear stresses $\tau\left(y_{i}, z_{i}\right), i=1,2,3$ at the position of the strain rosettes:

$$
\left[\begin{array}{l}
1 /\left(2 A_{m} t\right)-S_{z}\left(y_{1}, z_{1}\right) /\left(I_{z} t\right)-S_{y}\left(y_{1}, z_{1}\right) /\left(I_{y} t\right) \\
1 /\left(2 A_{m} t\right)-S_{z}\left(y_{2}, z_{2}\right) /\left(I_{z} t\right)-S_{y}\left(y_{2}, z_{2}\right) /\left(I_{y} t\right) \\
1 /\left(2 A_{m} t\right)-S_{z}\left(y_{3}, z_{3}\right) /\left(I_{z} t\right)-S_{y}\left(y_{3}, z_{3}\right) /\left(I_{y} t\right)
\end{array}\right] \cdot\left[\begin{array}{c}
M_{x} \\
V_{y} \\
V_{z}
\end{array}\right]=\left[\begin{array}{c}
\tau\left(y_{1}, z_{1}\right) \\
\tau\left(y_{2}, z_{2}\right) \\
\tau\left(y_{3}, z_{3}\right)
\end{array}\right]
$$

This system of linear equations can be solved for $V_{y}, V_{z}$ and $M_{x}$.

Equation (10), (11), (13) and (16) define a deterministic inverse model, which relates a strain state to stress resultants $\mathbf{R}=\left[N_{x}, V_{y}, V_{z}, M_{x}, M_{y}, M_{z}\right]^{T}$. In the following, this model is denoted by:

$$
\mathbf{R}=f\left(\boldsymbol{\varepsilon}_{1}, \boldsymbol{\varepsilon}_{2}, \boldsymbol{\varepsilon}_{3}, v, E\right)
$$

where $\mathbf{R}=\left[N_{x}, V_{y}, V_{z}, M_{x}, M_{y}, M_{z}\right]^{T}$ is the vector of stress resultants, $\boldsymbol{\varepsilon}_{i}=$ $\left[\varepsilon_{a, i}, \varepsilon_{b, i}, \varepsilon_{c, i}\right]^{T}$ is the vector of mechanical strains determined from strains measured by strain gauges $a, b$ and $c$ at position $i=1,2,3$. 


\subsection{Probabilistic Inverse Mechanical Model}

The reconstruction of stress resultants from the strain measurements is subject to uncertainty. Uncertainty is present in the model itself (model uncertainty), the material properties and the measurements. To model the uncertainty in the (inverse) mechanical model itself, a random variable $X_{\text {str }}$ with unit mean is introduced - in accordance with the probabilistic model code of the Joint Committee of Structural Safety (JCSS) [8] - which is multiplied to the model output, i.e.:

$$
\mathbf{R}=X_{s t r} \cdot f\left(\varepsilon_{1}, \boldsymbol{\varepsilon}_{2}, \boldsymbol{\varepsilon}_{3}, v, E\right)
$$

The uncertainty in the material properties is quantified by modelling the Young's modulus $E$ and the Poisson's ratio $v$ as random variables. This approach assumes that the spatial variability in the material properties is negligible. However, the model could be extended to account for the spatial variability by applying a random field approach.

The uncertainties in the mechanical strains $\varepsilon_{i}, i=1,2,3$ corresponding to the strains measured by the strain gauges at the positions of the different strain rosettes are modelled as described in Sect. 2.

\section{Numerical Example}

In the following, the approach presented in Sect. 2 and 3 is illustrated in a numerical example. First, the prior distribution of the mechanical strain $p^{\prime}\left(\varepsilon \mid \varepsilon_{M}, T\right)$ for a given measurement outcome $\varepsilon_{M}=60 \mu \mathrm{m} / \mathrm{m}$ and temperature $T=20{ }^{\circ} \mathrm{C}$ is constructed based on the process equation using MC simulation as described in Sect. 2.1 and the temperature-variation curve $\hat{\varepsilon}_{S}(T)$ given in Eq. (19). The simulation considers $10^{5}$ samples of the parameters $\mathbf{X}$ of the process equation, which are defined in In this numerical example, the posterior mean and standard deviation are $\mu_{\varepsilon}^{\prime \prime}=60.04 \mu \mathrm{m} / \mathrm{m}$ and $\sigma^{\prime \prime}{ }_{\varepsilon}=1.15 \mu \mathrm{m} / \mathrm{m}$. The probability density function (PDF) of the posterior mechanical strain is shown together with the likelihood function and the prior distribution in Fig. 3 .

Furth, a MC approach is applied to quantify the uncertainty in the reconstructed stress resultants. To this end, a constant strain state at the positions of the three strain rosettes is determined based on damage equivalent shear forces and bending moments, which were determined for the cross-section at the interface between the tower and the transition piece of an offshore wind turbine [12]. The computed strains are applied as the measurement outcomes. Strictly, an analysis of the posterior measurement uncertainty for each measured strain $\varepsilon_{M}$ and temperature T as described in Sect. 2 has to be performed. In this numerical example, however, we adopt a simplified approach, in which we assume that (a) the temperature is $\mathrm{T}=20^{\circ} \mathrm{C}$, (b) the posterior standard deviation $\sigma^{\prime \prime}{ }_{\varepsilon}$ determined above can be applied to quantify the uncertainty in the strain measurement regardless of the measured strain $\varepsilon_{M}$ and (c) individual measurements are statistically independent. Based on these assumptions, we generate $10^{5}$ independent and identical distributed (i.i.d.) samples of the mechanical strain $\varepsilon_{\mathrm{j}, \mathrm{i}}, \mathrm{j}=\mathrm{a}, \mathrm{b}, \mathrm{c}$ and $\mathrm{i}=1,2,3$ at the position of the strain rosettes from a normal distribution with mean equal to $\varepsilon_{\mathrm{M}, \mathrm{j}, \mathrm{i}}, \mathrm{j}=\mathrm{a}, \mathrm{b}, \mathrm{c}$ and $\mathrm{i}=1,2,3$ and standard deviation equal to $\sigma^{\prime \prime}{ }_{\varepsilon}$. In this way, we implicitly model the error in the strain measurements to be additive and normal distributed with zero mean 


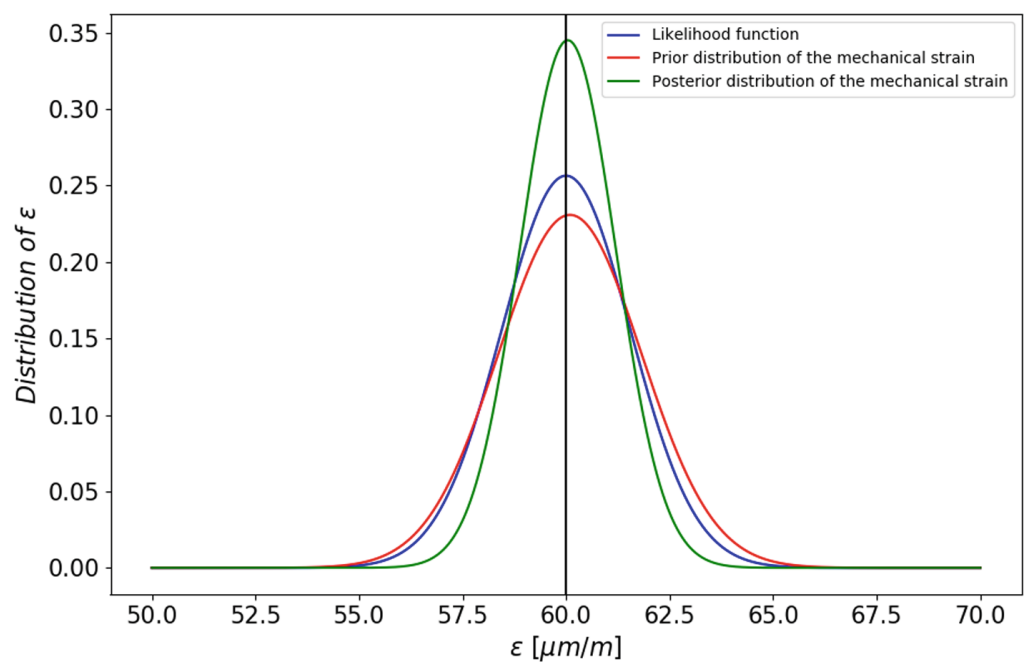

Fig. 3. Prior distribution $p^{\prime}\left(\varepsilon \mid \varepsilon_{M}, T\right)$ of $\varepsilon$, likelihood function $L\left(\varepsilon \mid \varepsilon_{M}, T\right)$ and posterior distribution $p^{\prime \prime}\left(\varepsilon \mid \varepsilon_{M}, T\right)$ of $\varepsilon$ for $\varepsilon_{M}=60 \mu \mathrm{m} / \mathrm{m}$ and $T=20^{\circ} \mathrm{C}$.

and standard deviation $\sigma^{\prime \prime}{ }_{\varepsilon}$. In addition, $10^{5}$ samples of the Young's modulus E, the Poisson's ratio $\nu$ and the model uncertainty $\mathrm{X}_{\text {str }}$ are generated. The probabilistic model of the parameters of the inverse mechanical model and the value of the angles $\alpha, \beta$ and $\gamma$ defining the orientation of the strain gauges in each strain rosette (see Fig. 1(b)) are given in Table 2.

It is assumed that $\varepsilon$ is normal distributed. The resulting prior mean and standard deviation of $\varepsilon$ are $\mu_{\varepsilon}^{\prime}=60.1 \mu \mathrm{m} / \mathrm{m}$ and $\sigma_{\varepsilon}^{\prime}=1.73 \mu \mathrm{m} / \mathrm{m}$.

$$
\hat{\varepsilon}_{T}(T)=-31.8+2.77 T-6.55 \cdot 10^{-2} T^{2}+3.28 \cdot 10^{-4} T^{3}-3.26 \cdot 10^{-7} T^{4}
$$

Second, a hypothetical laboratory experiment is performed as the basis for updating the prior measurement uncertainty. In the experiment, the mechanical strain and the temperature have fixed values $\varepsilon=60 \mu \mathrm{m} / \mathrm{m}$ and $T=20^{\circ} \mathrm{C}$. The mean and standard deviation of the measured strains are assumed to be $\mu_{\varepsilon_{M}}=60 \mu \mathrm{m} / \mathrm{m}$ and $\sigma_{\varepsilon_{M}}=1.55$ $\mu \mathrm{m} / \mathrm{m}$. As described in Sect. 2.2, the standard deviation $\sigma_{e}$ of the measurement error $e$ in Eq. (6) is equal to $\sigma_{\varepsilon_{M}}$ if $\varepsilon$ has a fixed value. Based on this, the likelihood function $L\left(\varepsilon \mid \varepsilon_{M}, T\right)$ is constructed as described in Sect. 2.2.

Third, the prior distribution $p^{\prime}\left(\varepsilon \mid \varepsilon_{M}, T\right)$ of the mechanical strain $\varepsilon$ is updated with $\varepsilon_{M}=60 \mu \mathrm{m} / \mathrm{m}$ and $T=20^{\circ} \mathrm{C}$ to the posterior distribution $p^{\prime \prime}\left(\varepsilon \mid \varepsilon_{M}, T\right)$ according to Eq. (9). Given that $p^{\prime}\left(\varepsilon \mid \varepsilon_{M}, T\right)$ and $L\left(\varepsilon \mid \varepsilon_{M}, T\right)$ have the functional form of a normal distribution, the posterior distribution $p^{\prime \prime}\left(\varepsilon \mid \varepsilon_{M}, T\right)$ also has this functional form. It can be shown that the posterior mean and variance $\mu_{\varepsilon}^{\prime \prime}$ and $\sigma_{\varepsilon}^{\prime \prime}$ are given by [11]:

$$
\mu_{\varepsilon}^{\prime \prime}=\frac{\mu_{\varepsilon}^{\prime} \sigma_{e}^{2}+\varepsilon_{M} \sigma_{\varepsilon}^{\prime 2}}{\sigma_{e}^{2}+\sigma_{\varepsilon}^{\prime 2}}
$$

$$
\sigma_{\varepsilon}^{\prime \prime}=\sqrt{\frac{\sigma_{e}^{2}{\sigma_{\varepsilon}^{\prime 2}}^{2}}{\sigma_{e}^{2}+{\sigma_{\varepsilon}^{\prime}}^{2}}}
$$


In this numerical example, the posterior mean and standard deviation are $\mu_{\varepsilon}^{\prime \prime}=60.04$ $\mu \mathrm{m} / \mathrm{m}$ and $\sigma_{\varepsilon}^{\prime \prime}=1.15 \mu \mathrm{m} / \mathrm{m}$. The probability density function (PDF) of the posterior mechanical strain is shown together with the likelihood function and the prior distribution in Table 1.

Table 1. Probabilistic and deterministic parameters of the process equation

\begin{tabular}{l|l|l|l|l}
\hline Parameter & Unit & Distribution & Values $^{\mathrm{a}}$ & References \\
\hline$B_{p}$ & $\mu \mathrm{m} / \mathrm{m}$ & normal & $\mu=0, \sigma=1$ & {$[2]$} \\
\hline$f_{a a}$ & - & uniform & $\mu=1, \sigma=1.73 \cdot 10^{-4}$ & {$[9,10]$} \\
\hline$f_{a z}$ & $\mu \mathrm{m} / \mathrm{m}$ & uniform & $\mu=0, \sigma=6.93 \cdot 10^{-1}$ & {$[9,10]$} \\
\hline$B_{S}$ & - & normal & $\mu=0, \sigma=4.38 \cdot 10^{-4}$ & {$[2]$} \\
\hline$f_{s, v}$ & - & normal & $\mu=0, \sigma=7 \cdot 10^{-3}$ & {$[9,10]$} \\
\hline$\alpha_{k}$ & $1 /{ }^{\circ} \mathrm{C}$ & normal & $\mu=0, \sigma=6.99 \cdot 10^{-3}$ & {$[9,10]$} \\
\hline$B_{T}$ & $\mu \mathrm{m} /(\mathrm{m} \mathrm{K})$ & normal & $\mu=0, \sigma=1.19$ & {$[9,10]$} \\
\hline$q$ & - & deterministic & $5 \cdot 10^{-4}$ & {$[5]$} \\
\hline$\nu_{0}$ & - & deterministic & 0.285 & {$[5]$} \\
\hline$\varepsilon_{q} / \varepsilon_{l}$ & - & deterministic & 0.3 & \\
\hline
\end{tabular}

${ }^{a} \mu$ and $\sigma$ are the mean and standard deviation of the corresponding parameter

Furth, a MC approach is applied to quantify the uncertainty in the reconstructed stress resultants. To this end, a constant strain state at the positions of the three strain rosettes is determined based on damage equivalent shear forces and bending moments, which were determined for the cross-section at the interface between the tower and the transition piece of an offshore wind turbine [12]. The computed strains are applied as the measurement outcomes. Strictly, an analysis of the posterior measurement uncertainty for each measured strain $\varepsilon_{M}$ and temperature $T$ as described in Sect. 2 has to be performed. In this numerical example, however, we adopt a simplified approach, in which we assume that (a) the temperature is $T=20^{\circ} \mathrm{C}$, (b) the posterior standard deviation $\sigma_{\varepsilon}^{\prime \prime}$ determined above can be applied to quantify the uncertainty in the strain measurement regardless of the measured strain $\varepsilon_{M}$ and (c) individual measurements are statistically independent. Based on these assumptions, we generate $10^{5}$ independent and identical distributed (i.i.d.) samples of the mechanical strain $\varepsilon_{j, i}, j=a, b, c$ and $i=1,2,3$ at the position of the strain rosettes from a normal distribution with mean equal to $\varepsilon_{M, j, i}$, $j=a, b, c$ and $i=1,2,3$ and standard deviation equal to $\sigma_{\varepsilon}^{\prime \prime}$. In this way, we implicitly model the error in the strain measurements to be additive and normal distributed with zero mean and standard deviation $\sigma_{\varepsilon}^{\prime \prime}$. In addition, $10^{5}$ samples of the Young's modulus $E$, the Poisson's ratio $v$ and the model uncertainty $X_{s t r}$ are generated. The probabilistic model of the parameters of the inverse mechanical model and the value of the angles $\alpha$, $\beta$ and $\gamma$ defining the orientation of the strain gauges in each strain rosette (see Fig. 1(b)) are given in Table 2 . 
Table 2. Probabilistic model of the parameters of the inverse mechanical model and values of the angles $\alpha, \beta$ and $\gamma$ defining the orientation of the strain gauges in each strain rosette.

\begin{tabular}{l|l|l|l|l}
\hline Parameter & Unit & Distribution & Values & References \\
\hline$E$ & $\mathrm{~N} / \mathrm{mm}^{2}$ & lognormal & $\mu=2.1 \cdot 10^{5}, \sigma=0.03 \cdot \mu$ & {$[8]$} \\
\hline$v$ & - & lognormal & $\mu=0.3, \sigma=0.03 \cdot \mu$ & {$[8]$} \\
\hline$X_{s t r}$ & - & lognormal & $\mu=1, \sigma=0.03 \cdot \mu$ & {$[13]$} \\
\hline$d$ & $\mathrm{~mm}$ & deterministic & 7000 & \\
\hline$t$ & $\mathrm{~mm}$ & deterministic & 70 & \\
\hline$\alpha$ & $\circ$ & deterministic & 45 & \\
\hline$\beta$ & $\circ$ & deterministic & 90 & \\
\hline$\gamma$ & $\circ$ & deterministic & 135 & \\
\hline
\end{tabular}

The histograms of the reconstructed bending moments $M_{y}$ and $M_{z}$ and shear forces $V_{y}$ and $V_{z}$ are shown in Fig. 4 (Note that the values of the reconstructed section forces and moments are here normalized by their mean value). In addition, Table 3 summarizes the coefficient of variations of the reconstructed section forces and moments, which are estimated based on their samples. The uncertainties in the bending moments are significantly lower than the uncertainties in the shear forces.
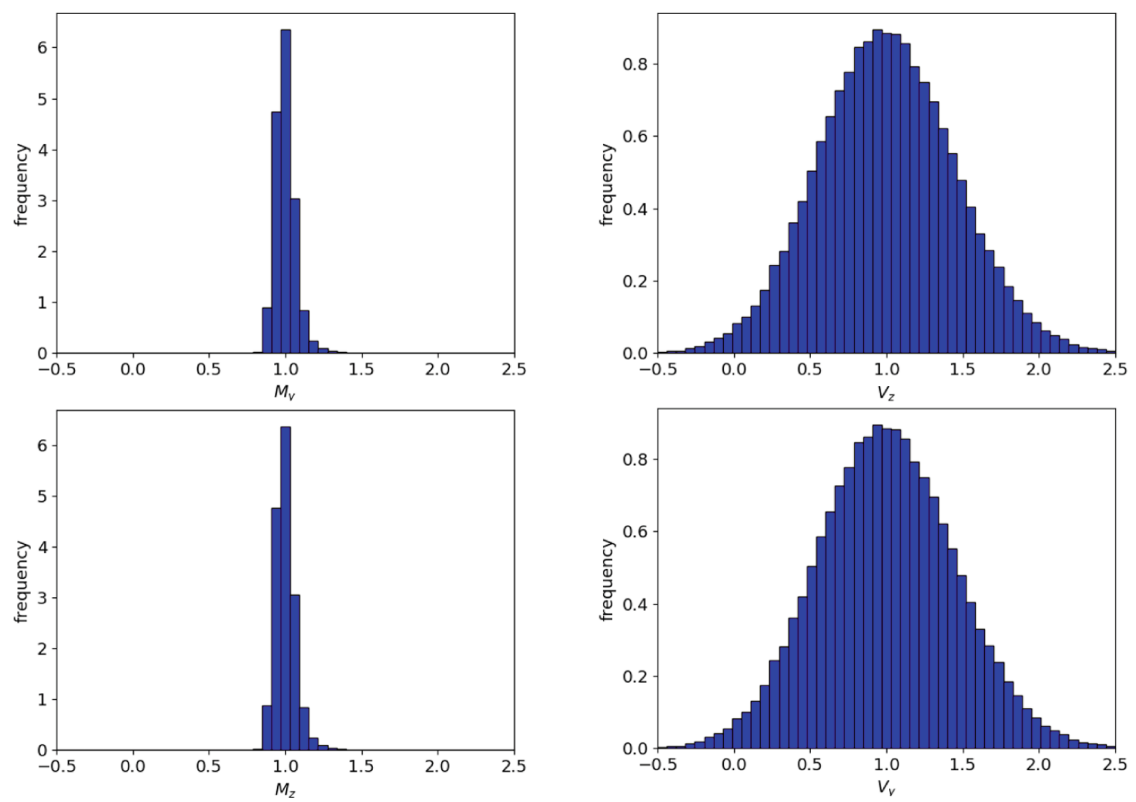

Fig. 4. Histograms of the reconstructed bending moments $M_{y}$ and $M_{z}$ and shear forces $V_{y}$ and $V_{z}$. The values of the reconstructed stress resultants are normalized by their mean values. 
Table 3. Coefficient of variation of the reconstructed stress resultants

\begin{tabular}{l|l|l|l}
\hline Shear forces & Coefficient of variation & Bending moments & Coefficient of variation \\
\hline$V_{y}$ & 0.37 & $M_{y}$ & 0.07 \\
\hline$V_{z}$ & 0.37 & $M_{z}$ & 0.07 \\
\hline
\end{tabular}

\section{Summary and Concluding Remarks}

In the first part of this contribution, a probabilistic model of strain measurements is formulated. The model is based on a process equation of the measurement process. It is applied to quantify a prior estimate of the measurement uncertainty. Bayesian methods are then applied to update the prior measurement uncertainty with outcomes of actual strain measurements. This approach takes all available information on the measurement process into account. The prior measurement uncertainty is based on the probabilistic formulation of the physical measurement process including model uncertainties associated with the applied physical model. The likelihood function is constructed based on a model of the observation-based measurement uncertainty and used to update the prior measurement uncertainty. By updating the prior measurement uncertainty, an improved estimate of the uncertainty in the strain measurement is obtained.

In the second part of the paper, an inverse mechanical model for reconstructing stress resultants in a cross-section of a wind turbine tower is presented. Finally, the methods for modelling strain measurements and the inverse mechanical model are applied in a numerical study considering a strain state derived by applying bending moments and shear forces to a turbine tower cross-section. In addition to the measurement uncertainties, the reconstruction is affected by the uncertainties in the material properties and the uncertainty in the mechanical model itself. The numerical study shows that the bending moments can be determined with the highest precision while the uncertainty in the shear forces is higher. The precision of the reconstruction depends mostly on the accuracy of the reconstructed stresses, which itself depend on the measurement uncertainty, its dependencies and the size of the measured strains.

Acknowledgment. This work was supported by the German Ministry for Economic Affairs and Energy (BMWi) through grant 03SX449Z.

\section{References}

1. Böttcher, J.: Handbuch Offshore-Windenergie: rechtliche, technische und wirtschaftliche Aspekte: Walter de Gruyter (2013)

2. Thöns, S.: Monitoring based condition assessment of offshore wind turbine support structures. IBK Bericht, vol. 345 (2012)

3. Thöns, S., Faber, M.H., Rücker, W.: On the utilization of monitoring data in an ultimate limit state reliability analysis, pp. 1762-1769 
4. Thöns, S., Faber, M.H., Rücker, W.: Life cycle cost optimized monitoring systems for offshore wind turbine structures. IRIS Industrial Safety and Life Cycle Engineering: Technologies/Standards/Applications, pp. 75-90 (2013)

5. Keil, S.: Beanspruchungsermittlung mit Dehnungsmessstreifen, Cuneus (1995)

6. Simoen, E., Papadimitriou, C., Lombaert, G.: On prediction error correlation in Bayesian model updating. J. Sound Vib. 332(18), 4136-4152 (2013)

7. Schnell, W., Gross, D., Hauger, W.: Technische Mechanik: Band 2: Elastostatik. SpringerVerlag, Heidelberg (2013)

8. JCSS Joint Committee on Structural Safety, "Probabilistic Model Code" 2001-2015

9. TML, TML WFLA-6-17 strain gauge test data (2008)

10. Verein Deutscher Ingenieure/Verband der Elektrotechnik Elektronik Informationstechnik, "VDI/VDE 2635 Part 1", Experimental structure analysis- Metallic bonded resistance strain gages - Characteristics and testing conditions, Verein Deutscher Ingenieure (2007)

11. Gelman, A., Carlin, J.B., Stern, H.S., Dunson, D.B., Vehtari, A., Rubin, D.B.: Bayesian Data Analysis. CRC Press, Cambridge (2013)

12. Wölfel Engineering GmbH + Co. KG, Fatigue Parameters of Arkona Becken OWF wind turbines for the MISO project (2020)

13. Tarp-Johansen, N., Madsen, P.H., Frandsen, S.T.: Calibration of partial safety factors for extreme loads on wind turbines

Open Access This chapter is licensed under the terms of the Creative Commons Attribution 4.0 International License (http://creativecommons.org/licenses/by/4.0/), which permits use, sharing, adaptation, distribution and reproduction in any medium or format, as long as you give appropriate credit to the original author(s) and the source, provide a link to the Creative Commons license and indicate if changes were made.

The images or other third party material in this chapter are included in the chapter's Creative Commons license, unless indicated otherwise in a credit line to the material. If material is not included in the chapter's Creative Commons license and your intended use is not permitted by statutory regulation or exceeds the permitted use, you will need to obtain permission directly from the copyright holder.

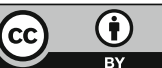

